\title{
Effectiveness of psychotherapeutic treatment of personality disorder ${ }^{\dagger}$
}

\author{
ANTHONY W. BATEMAN and PETER FONAGY
}

\author{
Background Psychiatrists have been \\ criticised for failing to develop adequate \\ treatment for personality disorder. \\ Psychotherapeutic treatments are \\ promising, but their effectiveness is \\ uncertain
}

\begin{abstract}
Aims To investigate the evidence for effectiveness of psychotherapeutic treatment for personality disorder.
\end{abstract}

Method Systematic literature review.

Results There is evidence for the effectiveness of psychotherapy for personality disorder. Problems of case identification, comorbidity, randomisation, specificity of treatment and outcome measurement are inadequately addressed. Authors mainly relied on cohort studies. Evidence neither suggests superiority of one type of therapy over another nor indicates which subgroups of patients should be offered psychotherapy as in-patient, day patient, or out-patient.

\section{Conclusions New research strategies are needed to show that personality change is both measurable and clinically meaningful. Effectiveness studies using randomised controlled designs are required. The literature suggests that effective treatment should be long-term, integrated, theoretically coherent and focused on compliance.}

\section{Declaration of interest None.}

In a riposte on mental health treatment in the UK (Times, 31 October 1998, p. 21) Jack Straw criticised psychiatrists for evading the treatment of personality disorder. Contemporary opinion is that there is little evidence of effective intervention. However, psychotherapeutic intervention is a promising treatment. The purpose of this article is to identify the evidence base for the effectiveness of psychotherapeutic treatment, to review critically some of the available data, to discuss obstacles to research which are revealed by the literature, to identify future research strategies and to summarise elements of treatment that may be useful for clinical practice.

\section{METHOD}

We searched the Medline and PsychLit databases, using both British and American spellings. We sought articles including the key terms 'personality disorder', 'treatment' and 'psychotherapy' and five types of specified psychosocial interventions, combined with eight terms referring to outcome data (e.g. 'controlled', 'outcome'). We reviewed 1814 abstracts and selected over 80 papers. The coverage was amended with a handsearch, the follow-up of bibliographies in identified studies and careful examination of review papers. The criteria used in selection were sample of patients with diagnoses of personality disorder, attempt at case identification, clear description of treatment, focus on Axis II problems, specified measures of outcome, mention of followup, and publication in the English language; papers that focused on the impact of Axis II diagnoses on the outcome of the treatment of Axis I disorder were excluded. Twenty-five primary reports of studies met these criteria. Because of limitations of space in this review, some older studies, and some which have been reported several times, were omitted.

\section{OUTCOME RESEARCH}

In view of its clinical utility and importance to the organisation of services, we have categorised outcome studies according to the context of treatment: in-patient, day patient or out-patient. They are further subdivided either into cohort studies or into studies that attempt to control for remission. In the latter category, most treatments are packages of care, and there are few randomised controlled trials (RCTs) of specific treatment. While there is overlap between the two types of study, those that focus primarily on long-term follow-up after a relatively non-specific intervention are included under the rubric of cohort studies.

\section{In-patient treatment}

Prolonged in-patient admission has been the mainstay of treatment of personality disorder.

\section{Cohort studies}

Most in-patient studies are prospective, using pre-test-post-test designs on one group. Tucker et al (1987) assessed the outcome after 1 and 2 years of 40 of 62 borderline patients treated for between 6 months and 1 year in a specialised unit. Treatment included individual, group and milieu therapies. Improvement in global function, reduction in self-destructive behaviour and suicide attempts, decreased use of hospitalisation, and the development of more constructive relationships were all found. Najavits \& Gunderson (1995) and Blatt \& Ford (1994) reported similar results. Copas et al (1984) retrospectively followed up 194 psychopathic patients after 3-5 years, and McGlashan (1986) retrospectively followed up 89 borderline patients after a mean of 15 years, both finding improvements in symptoms and in behaviour. Stone's (1993) report of up to 20 years' follow-up of 550 in-patients indicated that $66 \%$ of them were functioning well. Such studies fail to rule out other plausible alternative reasons for change, such as the passage of time or subsequent out-patient treatment. Rosser et al (1987) suggested, in a naturalistic 5-year followup of individuals receiving in-patient treatment at the Cassel Hospital in London, that caution should be exercised in ascribing any benefits observed to that treatment. Patients with borderline personality disorder (BPD) had a less favourable outcome than those with a combination of neurotic 
pathology, depression, high intelligence and lack of chronic out-patient history.

Longer-term follow-ups are hard to interpret because events, other therapies, etc. may have intervened; it could be argued that these studies really document the longterm course of the disorder.

\section{Controlled interventions}

Barley et al (1993) evaluated the effectiveness of dialectical behaviour therapy (DBT) for the treatment of BPD in an in-patient setting. DBT includes techniques at the level of behaviour (functional analysis), cognitions (e.g. skills training) and support (empathy, teaching management of trauma). They found that during and following implementation there was a significant fall in rates of parasuicide when compared to a period before implementation. There was no significant fall during an equivalent time period in another unit offering standard psychiatric care. However, it is not clear that the two groups of patients were comparable, the psychiatric care was not described and there was no randomisation of patients. Other structured inpatient treatments may have a similar effect, for example 'wellness and lifestyle groups', in which topics such as hobbies and fitness are discussed (Springer et al, 1996) or insight-oriented therapy (Liberman \& Eckman, 1981).

Dolan et al (1997) used a non-admitted comparison sample as a control to assess the effectiveness of specialist in-patient treatment on core symptoms of personality. This is one of the few studies to attempt assessment of syndromal change. They studied 137 patients, of whom 70 were admitted and 67 not admitted either for clinical or for financial reasons. One year after treatment there was significantly greater reduction in the core features of personality disorder on the Borderline Syndrome Index (BSI; Conte et al, 1980) in the treated group than in the non-admitted group. But in a UK study using the Personality Assessment Schedule (Tyrer et al, 1988) as the criterion measure, the BSI was found to lack validity and to be susceptible to distortion from current symptoms (Marlowe et al, 1996).

Limited data on cost suggests that in-patient admission may yield significant savings after completed treatment (Dolan et al, 1996), particularly in the use of criminal justice services in those with forensic histories. The true value of long-term in-patient treatment remains unclear, but in reviewing the literature on effectiveness, it is found that the patients likely to benefit are those showing: (a) substance misuse; (b) severe suicide risk; (c) forensic history; (d) transient difficulties in reality testing; (e) failure to respond to repeated short-term hospitalisation and out-patient intervention; and (f) evidence that destructive living and hopelessness has been incorporated into the personality.

\section{Day hospital treatment}

\section{Cohort studies}

Karterud et al (1992) studied prospectively 97 patients treated in a psychodynamically oriented day hospital, of whom $76 \%$ had an Axis II DSM-III-R diagnosis (American Psychiatric Association, 1987). After a mean treatment time of 6 months, the outcome on measures of global symptoms and overall mental health was found to be best for anxious-avoidant personality disorder, with only modest gains for BPD. Dick \& Woof (1986), using a similar programme, found that after 12 weeks of treatment a small subgroup of patients diagnosed retrospectively as having BPD increased their use of services, possibly indicating that a longer term of treatment was necessary for this group.

There is little evidence of specificity of treatment programmes. A feminist, psychodynamically informed programme with a socio-political dimension was effective in reducing symptoms and health service usage in 31 patients with personality disorder treated in day and semi-residential facilities. Gains were sustained over a 2year follow-up (Krawitz, 1997). Use of a socio-political dimension may be highly pertinent, given the breadth of social adaptational difficulties of most patients.

\section{Controlled studies}

In a prospective study, using a design of treatment $v$. control (delayed treatment), Piper et al (1993) found significant effects of 18 weeks of day hospital treatment in 79 patients with both affective disorder and long-standing personality disorder. Interpersonal functioning, symptoms, selfesteem, life satisfaction and defensive functioning all improved after 4 months of treatment when compared with the control group, and gains were maintained at the 8-month follow-up.
Recently, Bateman \& Fonagy (1999), in a controlled trial of 38 patients with BPD, randomly allocated to a psychoanalytically informed day hospital or to treatment as usual (TAU), reported a substantial reduction in parasuicidal behaviour, self-harm and hospitalisation over an 18-month period of treatment. The severity of selfreported mood and psychiatric symptoms also improved substantially relative to the control group. Drop-out was low (12\%). Improvement occurred later in treatment, emphasising that admission to day hospital needs to be for a relatively long term. Follow-up data are not yet available, nor is it clear which of a number of potentially effective components (the therapy, the milieu, or contact with staff) may have been responsible for the improvements.

There are no data to suggest that the mere inclusion of psychotherapy within a day hospital is sufficient to ensure good outcome. All studies use an integrated and organised treatment programme within a singular, coherent (and to the patients understandable) system. Only such integrated programmes, with clinicians with various tasks and functions working to the same strategic goals, permit patients with severe problems in understanding human motives (Fonagy, 1998) to feel sufficiently safe to engage effectively with the treatment.

Since there are no comparative outcome studies of different contexts of treatment, clinical impressions generated in the course of this review, rather than empirical data, are the only guide to indications for day hospital treatment. These are similar to those for in-patient treatment.

\section{Out-patient treatment}

\section{Cohort studies}

Data on both cognitive-behavioural (CBT) and psychodynamic treatment come primarily either from single-case series or from theory-oriented methods, which makes it difficult to generalise the results. Older studies of psychodynamic treatment reporting long-term follow-up suggest that some patients do well while others, particularly those with comorbidity, do badly (Wallerstein, 1986). More recently, Stevenson \& Meares $(1992,1999)$ reported on 48 patients with BPD treated with psychoanalytic psychotherapy twice a week for one year. Significant improvements in the number of episodes of self-harm and violence, length of hospital admissions, 
and other measures were observed in the 30 patients who completed therapy; and $30 \%$ of patients no longer fulfilled the criteria for BPD at the end of treatment. Improvement was maintained over 1 year, and continued over 5 years, with substantial saving in health care costs.

Davidson \& Tyrer (1996) found important changes in maladaptive behavioural and cognitive patterns after a 10 -week course of CBT in 12 out-patients with antisocial disorder or BPD. Turkat $\&$ Maisto (1985) formulated 35 patients from a cognitive-behavioural perspective and, using a single-case design, tested their formulations. Of the 16 cases for which outcome data were available, only four had a positive outcome.

The results of these studies must be treated with circumspection. There were no independent ratings, no comparison groups, and no discussion of the possibility of improvement over time.

\section{Controlled studies}

In one of the few randomised controlled trials of out-patient treatment of BPD, Linehan and colleagues (1991) show that DBT was partially effective in helping women patients with BPD. Therapy was conducted weekly, and offered both individually and in groups over one year. Interventions received by patients under the TAU regime were not controlled. Twentytwo women patients were assigned to DBT and 22 to the control group. Assessment was carried out during, and at the end of, therapy, and again after 1 year follow-up (Linehan et al, 1993). The drop-out rate was low (16\%). Control patients were significantly more likely to make suicide attempts, spent longer as inpatients over the year of treatment, and were more likely to drop out of those therapies to which they were assigned. However there were no differences between groups on measures of depression, hopelessness or reasons for living. Follow-up at 1 year found no differences between groups.

Only recently have controlled studies of out-patient psychoanalytic therapy been instigated. Clarkin et al (1999) have reported preliminary data on a small sample of 10 patients with BPD treated for 1 year. A number showed significant improvements. Patients with antisocial features seem particularly likely to do badly. A comparative trial of relationship management group therapy and individual dynamic treatment for BPD indicated, at the end of treatment and at follow-up, that these were equally effective (Monroe-Blum \& Marziali, 1995), suggesting a need for cost-benefit analysis to be included in future research.

Results from controlled studies of avoidant personality disorder suggest that both dynamically oriented and behavioural packages are useful (see Roth \& Fonagy, 1996, for review). But many patients do not achieve normal functioning. Alden \& Capreol (1993) suggest that patients with avoidant personality disorder are either distrustful and angry, or underassertive. In a trial of 76 patients, angry patients benefited from exposure but not from skills training, while the unassertive improved with both. Subdividing diagnostic categories helps to focus treatment more effectively, as may recognising interactions between aptitude and treatment. 'Deconstructing' descriptions of personality disorders into personality style and disordered function components may be appropriate for future research. Beutler et al (1991) suggest that clients who are reactant (resistant) benefit more from non-directive therapy or paradoxical interventions than from cognitivebehavioural interventions.

\section{Summary}

In summarising studies of out-patient treatment, it should be noted that there is relatively little compelling evidence that individuals with personality disorders and low levels of functioning can be successfully treated on an out-patient basis, although they may be supported. The best evidence so far from Linehan et al's (1991) DBT trial is relatively disappointing in terms of long-term outcome. There are preliminary indications that individuals with predominantly avoidant personality disorders may be helped by either dynamic or behavioural methods.

\section{PROBLEMS OF RESEARCH}

This literature review revealed several serious problems that need to be addressed if future research is to be fruitful.

\section{Case identification}

There are problems of case identification in this literature, since subjects may be grouped either by categories based on DSM-IV and ICD-10 (World Health Organization, 1992), or by a theory- oriented method. Both methods present problems. There is no predictive value to categories. Forming three clusters of oddeccentric, impulsive-erratic, and anxiousavoidant has only face validity. No cohort studies have been carried out to confirm the stability of such groupings (Mulder $\&$ Joyce, 1997). There is poor cross-classificatory reliability (Sara et al, 1996), making comparison of research findings difficult. If this method is to be improved, future research may have to identify personality disorder from the perspectives of both the patient and an informant (Zimmerman, 1994). It is not possible to generalise the findings of theory-oriented evaluations (see, e.g. Beck \& Freeman, 1990, for cognitive; Benjamin \& Benjamin, 1994, for interpersonal; and Kernberg, 1984, for a psychoanalytic perspective), and their use to compare the relative effectiveness of alternative clinical approaches is problematic. But they offer a practical way of refining conceptualisations of specific personality disorders.

Dimensional approaches are of limited value in clinical outcome studies. There is agreement neither on traits nor on whether they represent extremes of normal personality or are qualitatively different, and the two are often conflated by assessing traits which are abnormal in quantity (e.g. sociability) alongside personality dispositions which are unlikely to be found in nonclinical subjects (e.g. self-mutilation). Recently, a method for revising and measuring Axis II categories has been developed that is both psychometrically and clinically meaningful (Westen \& Shedler, 1999). This offers potential for future research.

\section{Comorbidity}

Often several personality disorders are reported in one individual, and interaction between the personality disorder and Axis I diagnosis could result in either an exaggeration or an obscuring of a genuine treatment effect. In the majority of studies, measured change in personality may be an artefact related to improvement in symptoms, which itself may be due to a change in the manifestation of the personality disorder, rather than improvement: for example, a patient with BPD may avoid intense relationships and so show fewer symptoms. In general, studies confound treatment effects related to personality change with improvement in symptoms. 


\section{Randomisation and control}

Problems of implementing RCTs for assessing the efficacy of psychotherapeutic treatments have been discussed elsewhere (Roth \& Fonagy, 1996). But problems specific to personality disorder may account for the dearth of controlled studies identified for this review. First, keeping an RCT running for a long time period with an adequate number of patients is expensive and a formidable logistical problem. Attrition rates were high in many studies, due to the chaotic lifestyles of the subjects and their social mobility, although this can be ameliorated (Linehan et al, 1991; Stevenson \& Meares, 1992; Bateman \& Fonagy, 1999). The therapist's investment in the initial treatment contract and maintenance of an alliance improves compliance (Yeamans $e t$ al, 1994). Recent studies suggest that younger patients who are initially hostile are the most likely to withdraw (Smith $e t$ al, 1995). Second, intercurrent treatment is inevitable and confounded all long-term follow-ups. Third, patients' expectations are an important factor for therapy outcome (Horowitz et al, 1993): randomisation to different treatments may lead to allocations incongruent with these expectations, and this may be particularly problematic for patients whose lack of flexibility is almost a defining feature of their disorder (Bleiberg, 1994). Waiting-list controls cannot be used to control for change over long periods of time, and so are of limited use. The most stringent control group without ethical problems is TAU which should be used in future, even though heterogeneity of intervention and differential responsiveness within groups may obscure results.

\section{Specificity of psychotherapies}

Evaluation of outcomes is hampered by the lack of specificity in psychotherapeutic approaches. There is so much variance within each treatment, and overlap between treatments, that differential treatment effects are likely to be masked (Goldfried \& Wolfe, 1998). Thus comparative outcome studies are unlikely to produce a yield commensurate with their effort. In long-term therapy, practitioners make complex choices in selecting interventions that take account of both behavioural and dynamic factors. In order to enhance specificity and to assess fidelity of application, researchers have 'manualised' treatments. These include psychoanalytic psychotherapy (Kernberg et al, 1989; Clarkin et al, 1999), DBT (Linehan, 1993), and object relations/interpersonal approaches (Dawson, 1988). The manual for cognitive-analytic therapy is as yet untested (Ryle, 1997).

\section{OUTCOME MEASUREMENT AND FOLLOW-UP}

The comparability of outcome measures used in recent studies is impressive. Most assess symptoms, behaviour, social adjustment and psychiatric status, but rarely actual change in syndromes. It is necessary to agree on basic outcome measures, which itself requires a consensus on treatment goals. In studies of offenders with personality disorder, reconviction is the most commonly used outcome measure, although there is no agreement on its appropriateness (Dolan \& Coid, 1993). Instruments should reflect the perspectives of both the patient and the observer (therapist, independent rater, or informant from the patient's social network). Without reliable case identification, measuring personality change itself is compromised, and most studies rely on improvements in symptoms and behaviour as indicators of underlying structural change. Some (e.g. Stevenson \& Meares, 1992; Monson et al, 1995; Dolan et al, 1997) use reduction in the number of positive criteria as an indicator of change in syndromes, and there may be a relationship between duration of treatment and the reduction in positive symptoms of personality disorder (Perry, 1993). Better ways are needed, and instruments such as the Adult Attachment Interview (Main et al, 1985) may be useful in the future.

The necessity for long-term follow-up in studies of the effectiveness of treatment of personality disorder is widely acknowledged. The outcome of therapy may not be fully evident at discharge (Hogland et al, 1993).

\section{DISCUSSION}

In the light of the considerable problems which still exist in conceptualising and defining personality disorder, in separating it from other mental disorders and in designing treatment trials with adequate internal and external validity, it is not surprising that our knowledge of effective psychological treatments is rudimentary. Despite an attempt at a meta-analysis of outcome (Perry et al, 1999), the research does not meet the robust requirements of an empirically based clinical discipline: failing to study clearly defined populations, to define treatment carefully and to assess its specificity, to ensure treatment is superior to no treatment or TAU, and to demonstrate that the treatment has an effect on the personality rather than merely causing a change in symptoms. Effective treatment protocols are few in number and remain largely untested. As the vast majority of studies are uncontrolled and independent raters are never used, caution is essential in interpreting the findings. None the less, studies consistently demonstrate modest gains associated with relatively high doses of treatment. There is encouraging evidence that these gains are cost-effective (Gabbard et al, 1997), particularly in terms of savings in health care costs. Future studies should show that personality change is both measurable and clinically meaningful, be based on random allocation, carefully define treatment goals, include adequate follow-up, and address the cost-effectiveness of such treatment compared with alternative interventions.

\section{Effective ingredients of treatment}

Treatments shown to be moderately effective have certain common features. They tend: (a) to be well structured; (b) to devote considerable effort to enhancing compliance; (c) to have a clear focus, whether that focus is a problem type of behaviour such as self-harm or an aspect of interpersonal relationship patterns; (d) to be theoretically highly coherent to both therapist and patient, sometimes deliberately omitting information incompatible with the theory; (e) to be relatively long term; (f) to encourage a powerful attachment relationship between therapist and patient, enabling the therapist to adopt a relatively active rather than a passive stance; and (g) to be well integrated with other services available to the patient. While some of these features may be those of a successful research study rather than of a successful therapy, the manner in which clinical treatment protocols are constructed and delivered is probably as important in the success of treatment as the specific, theoretically driven intervention itself.

\section{Pathway to effectiveness}

One way of interpreting these observations might be that part of the benefit which 
individuals with personality disorder derive from treatment comes through experience of being involved in a carefully considered, well structured and coherent interpersonal endeavour. What may be helpful is the internalisation of a thoughtfully developed structure, the understanding of the interrelationship of different reliably identifiable components, the causal interdependence of specific ideas and actions, the constructive interactions of professionals, and above all the experience of being the subject of reliable, coherent and rational thinking. Social and personal experiences such as these are not specific to any treatment modality but, rather, indicate the level of seriousness and the degree of commitment with which teams of professionals approach the problem of caring for this group, who may be argued on empirical grounds to have been deprived of exactly such consideration and commitment during their early development and quite frequently throughout their later life (see review by Zanarini \& Frankenburg, 1997). While this suggestion is speculative, it may also be helpful in distinguishing successful from unsuccessful interventions and pointing the way to the creation of more efficacious protocols in the future.

\section{REFERENCES}

Alden, L. E. \& Capreol, M. J. (1993) Avoidant personality disorder: interpersonal problems as predictors of treatment response. Behavioural Therapy, 24, 357-376.

American Psychiatric Association (1987) Diagnostic and Statistical Manual of Mental Disorders (3rd edn, revised) (DSM-III-R). Washington, DC: APA

Barley, W. D., Buie, S. E., Peterson, E.W., et al (1993) Development of an in-patient cognitive-behavioural treatment program for borderline personality disorder. Journal of Personality Disorders, 7, 232-240.

Bateman, A.W. \& Fonagy, P. (1999) The effectiveness of partial hospitalisation in the treatment of borderline personality disorder: a randomised controlled trial. American journal of Psychiatry, 156, 1563-1569.

Beck, A. T. \& Freeman, A. (1990) Cognitive Therapy of Personality Disorders. New York: Guilford.

Benjamin, L. \& Benjamin, R. (1994) Application of contextual therapy to the treatment of multiple personality disorder. Dissociation Progress in the Dissociative Disorders, 7, 12-22.

Beutler, L. E., Engle, D., Mohr, D., et al (1991) Predictors of differential response to cognitive, experiential, and self-directed psychotherapeutic procedures. Journal of Consulting \& Clinical Psychology, 59, 333-340.

Blatt, S. J. \& Ford, R. Q. (1994) Therapeutic Change: An Object Relations Perspective. New York: Plenum.

Bleiberg, E. (1994) Borderline disorders in children and adolescents: the concept, the diagnosis, and the controversies. Bulletin of the Menninger Clinic, 58, 169-196.

Clarkin, J. F, Kernberg, O. F. \& Yeomans, F. (1999) Transference-Focused Psychotherapy for Borderline Personality Disorder Patients. New York: Guilford.

Conte, H., Putchik, R., Karasu, T., et al (1980) A self-report borderline scale: discriminative validity and preliminary norms. Journal of Nervous and Mental Disease, 168, 428-435.

Copas, J. B., O'Brian, M. O., Roberts, J., et al (1984) Treatment outcome in personality disorder: the effect of social, psychological and behavioural variables. Personality \& Individual Differences, 5, 565-573.

Davidson, K. M. \& Tyrer, P. (1996) Cognitive therapy for antisocial and borderline personality disorders: single case study series. British Journal of Clinical Psychology, 35, 413-429.

Dawson, D. (1988) Treatment of the borderline patient: relationship management. Canadian Journal of Psychiatry, 33, 370-374.

Dick, B. M. \& Wooff, K. (1986) An evaluation of a time-limited programme of dynamic group psychotherapy. British Journal of Psychiatry, 148, 159-164.

Dolan, B. \& Coid, J. (1993) Psychopathic and Antisocial Personality Disorders: Treatment and Research Issues. London: Gaskell.

_ ,Warren, F. M., Menzies, D., et al (1996) Costoffset following specialist treatment of severe personality disorder. Psychiatric Bulletin, 20, 413-417.

_, _ \& Norton, K. (1997) Change in borderline symptoms one year after therapeutic community treatment for severe personality disorder. British Journal of Psychiatry, I7I, 274-279.

Fonagy, P. (1998) An attachment theory approach to treatment of the difficult patient. Bulletin of the Menninger Clinic, 62, 147-169.

Gabbard, G. O., Lazar, S. G., Hornberger, J., et al (1997) The economic impact of psychotherapy: a review. American Journal of Psychiatry, 154, 147-155.

Goldfried, M. R. \& Wolfe, B. E. (1998) Toward a more clinically valid approach to therapy research. Journal of Consulting \& Clinical Psychology, 66, 143-150.

Hogland, P. (1993) Personality disorders and long-term outcome after brief psychodynamic psychotherapy. Journal of Personality Disorders, 7, 168-181.

Horowitz, L. M., Rosenberg, S. E. \& Bartholomew, K. (1993) Interpersonal problems, attachment styles and outcome in brief dynamic therapy. Journal of Consulting \& Clinical Psychology, 6I, 549-560.

Karterud, S., Vaglum, S., Friis, S., et al (1992) Day hospital therapeutic community treatment for patients with personality disorders: an empirical evaluation of the containment function. Journal of Nervous \& Mental Disease, 180, 238-243.

Kernberg, O. F. (1984) Severe Personality Disorders: Psychotherapeutic Strategies. New Haven, CT: Yale University Press.

_ , Selzer, M. A., Koenigsberg, H. W., et al (1989) Psychodynamic Psychotherapy of Borderline Patients. New York: Basic Books.

Krawitz, R. (1997) A prospective psychotherapy outcome study. Australian \& New Zealand Journal of Psychiatry, 3I, 465-473.

Liberman, R. P. \& Eckman, T. (198I) Behavior therapy vs insight oriented therapy for repeated suicide attempters. Archives of General Psychiatry, 38, 1126-1130.
Linehan, M. M. (1993) The Skills Training Manual for Treating Borderline Personality Disorder. New York: Guilford.

_ , Armstrong, H. E., Suarez, A., et al (199I)

Cognitive-behavioural treatment of chronically parasuicidal borderline patients. Archives of General Psychiatry, 48, 1060-1064.

\section{_ , Heard, H. L. \& Armstrong, H. E. (1993)}

Naturalistic follow-up of a behavioral treatment for chronically parasuicidal borderline patients. Archives of General Psychiatry, 50, 971-974.

Main, M., Kaplan, N. \& Cassidy, J. (1985) Security in Infancy, Childhood and Adulthood: A Move to the Level of Representation. Monographs of the Society for Research in Child Development, no. 50. Chicago, IL: Chicago University Press.

Marlowe, M. J., O'Neill-Byrne, K., Lowe-Ponsford, F., et al (1996) The Borderline Syndrome Index: a validation study using the personality assessment schedule. British Journal of Psychiatry, $\mathbf{1 6 8}$. $72-75$.

McGlashan, T. (1986) The Chestnut Lodge Follow-up Study. III: Long-term outcome of borderline personalities. Archives of General Psychiatry, 43, 20-30.

Monroe-Blum, H. \& Marziali, E. (1995) A controlled trial of short-term group treatment of borderline personality disorder. Journal of Personality Disorders, $\mathbf{9}$ 190-198.

Monson, J.T., Odland, T. \& Eilertsen, D. E. (1995)

Personality disorders: changes and stability after intensive psychotherapy focusing on affect consciousness. Psychotherapy Research, 5, 33-48.

Mulder, R. T. \& Joyce, P. R. (1997) Temperament and the structure of personality disorder symptoms. Psychological Medicine, 27, 91-106.

Najavits, L. M. \& Gunderson, J. G. (1995) Improvements in borderline personality disorder in a 3 year prospective outcome study. Comprehensive Psychiatry, 36, 296-302.

Perry, J. C. (1993) Longitudinal studies of personality disorders. Journal of Personality Disorders, 7 (suppl.), 63-85.

_ , Banon, E. \& lanni, F. (1999) Effectiveness of psychotherapy for personality disorders. American Journal of Psychiatry, 156, 1312-1321.

Piper, W. E., Joyce, A. S., Azim, H. F. A., et al (1993) Patient characteristics and success in day treatment. Journal of Nervous \& Mental Disease, 182, 38I-386.

Rosser, R., Birch, S., Bond, H., et al (1987) Five year follow-up of patients treated with in-patient psychotherapy at the Cassel Hospital for Nervous Diseases. Journal of the Royal Society of Medicine, $\mathbf{8 0}$ 549-555.

Roth, A. \& Fonagy, P. (eds) (1996) What Works for Whom? A Critical Review of Psychotherapy Research. New York: Guilford.

Ryle, A. (1997) Cognitive Analytic Therapy and Borderline Personality Disorder: The Model and the Method. Chichester: Wiley.

Sara, G., Raven, P. \& Mann, A. (1996) A comparison of DSM-III-R and ICD-10 personality disorder criteria in an outpatient population. Psychological Medicine, 26, $|5|-160$.

Smith, T. E., Koenigsberg, H.W., Yeomans, F. E., et al (1995) Predictors of dropout in psychodynamic psychotherapy of borderline personality disorder. Journal of Psychotherapy Practice and Research, 4 205-213. 
Springer, T., Lohr, N. E., Buchtel, H. A., et al (1996) A preliminary report of short-term cognitive-behavioural group therapy for in-patients with personality disorders. Journal of Psychotherapy Practice and Research, 5, 57-7l.

Stevenson, J. \& Meares, R. (1992) An outcome study of psychotherapy for patients with borderline personality disorder. American Journal of Psychiatry, 149, 358-362.

_ \& _ (1999) Psychotherapy with borderline patients. II: A preliminary cost-benefit study. Australian \& New Zealand Journal of Psychiatry, 33, 473-477.

Stone, M. H. (1993) Long-term outcome in personality disorders. British Journal of Psychiatry, 162, 299-313.

Tucker, L., Bauer, S. F., Wagner, S., et al (1987) Longterm hospital treatment of borderline patients: a descriptive outcome study. American Journal of Psychiatry, 144, 1443-1448.

Turkat, I. D. \& Maisto, S. A. (1985) Personality disorders: application of the experimental method to the formulation and modification of personality disorders. In Clinical Handbook of Psychological Disorders: A Step-by-step Treatment Manual (ed. D. H. Barlow). New York: Guilford.

\section{Tyrer, P., Ferguson, B. \& Alexander, J. (1988)}

Personality assessment schedule. In Personality Disorder: Diagnosis, Management and Course (ed. P. Tyrer). Bristol: John Wright.

Wallerstein, R. S. (1986) Forty-two Lives in Treatment: A Study of Psychoanalysis and Psychotherapy. New York: Guilford.

Westen, D. \& Shedler, J. (1999) Revising and assessing Axis II. Part I: Developing a clinically meaningful and empirically valid assessment method. American journal of Psychiatry, I56, 258-272.

World Health Organization (1992) The ICD-10 Classification of Mental and Behavioural Disorders. Geneva: WHO

Yeamans, F. E., Gutfreund, J., Selzer, M. A., et al (1994) Factors related to drop-outs by borderline patients: treatment contract and therapeutic alliance. Journal of Psychotherapy Practice and Research, 3, 16-24.

Zanarini, M. C. \& Frankenburg, F. R. (1997) Pathways to the development of borderline personality disorder. Journal of Personality Disorders, II, 93-104.

Zimmerman, M. (1994) Diagnosing personality disorders. A review of issues and research methods. Archives of General Psychiatry, 5I, 225-245.

\section{CLINICAL IMPLICATIONS}

- There is evidence for the effectiveness of psychotherapy for treatment of personality disorder. Most evidence supports long-term cognitive-behavioural approaches and psychodynamic orientations with Cluster B patients. These approaches have generalised treatment protocols, which can be applied on an outpatient basis.

- Treatments need to be well structured and packaged, to have a clear focus, to be theoretically coherent and to be well integrated with other services.

- In-patient, day patient and out-patient treatment have been shown to be useful. In-patient treatment should be reserved for patients showing substance misuse, severe suicide risk, a forensic history and difficulties in reality testing, and for those that have failed in repeated short-term hospital admission and out-patient intervention.

\section{LIMITATIONS}

- Most of the evidence is either descriptive or qualitative, relying more on cohort studies than controlled trials. Non-English-language journals were not covered by this review.

Research is limited by problems of case identification, comorbidity, specificity of treatment, agreed measures of outcome and adequate follow-up.

- The field is not yet ready for more systematic quantitative reviews, including metaanalysis.
ANTHONY W. BATEMAN, FRCPsych, Halliwick Psychotherapy Unit, St Ann's Hospital, London; PETER FONAGY, PhD, Psychoanalysis Unit, University College London

Correspondence: Dr A.W. Bateman, Halliwick Psychotherapy Department, Haringey Healthcare NHS Trust, St Ann's Hospital, St Ann's Road, London NI5 3TH

(First received 18 December 1998, final revision 3 December 1999, accepted 7 December 1999) 